Tejada, E., Garay, U., Romero, A. y Bilbao, N. (2021). El bullying desde el punto de vista del acosador: análisis̄ y procedimiento. Revista de Investigación Educativa, 39(2), 373-390.

DOI: http://dx.doi.org/10.6018/rie.422671

\title{
El bullying desde el punto de vista del acosador: análisis y procedimiento
}

\section{Bullying from the bully's point of view: analysis and procedure}

\author{
Eneko Tejada, Urtza Garay, Ainara Romero y Naiara Bilbao \\ Departamento de Didáctica y Organización Escolar. Facultad de Educación de Bilbao. \\ Universidad del País Vasco/Euskal Herriko Unibertsitatea
}

\begin{abstract}
Resumen
Los centros de enseñanza poseen numerosos instrumentos para prevenir el acoso escolar, pero existe una gran escasez de medios para detenerlo desde una perspectiva pedagógica, cuando ya se ha originado. Esta investigación tiene por objeto diseñar un procedimiento eficaz que permita detener el bullying en esta situación y la muestra la conforman seis estudiantes de Educación Secundaria Obligatoria (ESO) identificados como acosadores escolares. Para el desarrollo de este estudio se ha adoptado una metodología de investigación acción, lo que ha facilitado el diseño de una herramienta anti-bullying, su implementación, la observación de cómo funciona y la valoración acerca de su incidencia y eficacia. El análisis de los datos ha sido cuantitativo y cualitativo, lo que ha permitido observar el efecto de las actividades en las conductas acosadoras en las seis sesiones llevadas a cabo y conocer el nivel de comprensión y tipo de pensamiento por el que pasa el acosador antes de dejar de agredir. De los resultados se extrae un procedimiento anti-bullying avanzado que constituye una propuesta válida para la intervención con el alumnado acosador y que contribuye a detener las conductas de acoso escolar.
\end{abstract}

Palabras clave: Bullying; agresor; intervención; niveles de comprensión.

Correspondencia: Eneko Tejada Garitano, eneko.tejada@ehu.eus, Facultad de Educación de Bilbao, Sarriena $\mathrm{s} / \mathrm{n}$. 


\begin{abstract}
Schools have many tools to prevent bullying, but once it has appeared, there is a lack of pedagogical strategies to stop it. This research aims to design an effective procedure to stop bullying by analyzing a sample of six students from Secondary School who have been identified as bullies. In order to carry out this study, an action research methodology has been adopted which has allowed the design of an anti-bullying tool, its implementation, the observation of how it works and the assessment of its incidence and effectiveness. Both quantitative and qualitative analyses of the data have been performed during six sessions in order to observe the effect of the activities on the bullying behaviors, as well as the level of understanding and the type of thinking that the bully goes through before stopping the attack. The results have led to the design of an advanced anti-bullying procedure which constitutes a valid proposal for intervention with the bullying students and contributes to stopping bullying behaviors.

Keywords: Bullying; aggressor; intervention; levels of understanding.
\end{abstract}

\title{
Introducción
}

El Bullying, también conocido como acoso escolar o maltrato entre iguales, es un tipo de agresión o violencia específica que sucede en el ámbito educativo. Fundamentalmente, se trata de un fenómeno que hace referencia al conjunto de acciones dañinas que uno o varios sujetos causan de forma consciente, recurrente y mantenida en el tiempo (Olweus, 2013; Smith, 2016). Diferentes estudios señalan que el objetivo del alumnado que comete bullying es victimizar a una tercera persona que se encuentra en situación de indefensión para producirle un daño psicológico, social y/o físico (Ruiz, et al., 2015; Volk, Dane \& Marini, 2014). Para entender el fenómeno bullying y el motivo por el que se origina, se deben analizar factores tan complejos como el contexto social y cultural de donde se está produciendo, así como el medio familiar de la víctima y de los estudiantes que participan de forma directa e indirecta en las agresiones, ya que se trata de un proceso socioecológico (Cerezo, et al., 2015; Swearer \& Hymel, 2015; Lyng, 2018). Por esta razón, los centros educativos deberían hacer frente al acoso escolar desde una perspectiva psicosocial e implicar a toda la comunidad educativa; profesorado, alumnado y familias (Della Cioppa, O'Neil \& Craig, 2015; Garaigordobil, 2017).

\section{Acoso Escolar}

En la actualidad las instituciones educativas tienen a su disposición numerosos recursos para su alumnado que contribuyen a la erradicación del bullying desde una perspectiva preventiva primaria, es decir, de forma universal e integral antes de que suceda el acoso. Entre todos los instrumentos destaca fundamentalmente la exitosa iniciativa anti-bullying KiVa desarrollada por la Universidad de Turku (Granero \& Manzano, 2018, Nocentini \& Menesini, 2016), la cual permite a los centros educativos luchar contra el acoso escolar adelantándose al problema y desarrollar de forma estratégica la convivencia (Saarento, Boulton \& Salmivalli, 2015). Los colegios e institutos también utilizan otro tipo de programas que se orientan a intervenir exclusivamente sobre el alumnado que 
se encuentra en situación de riesgo, es decir, su actuación se limita al contexto de los agresores y víctimas potenciales (Bonell, Allen, Warren et al., 2018; Mytton et al., 2006).

Para las situaciones en que ya se ha producido el acoso apenas hay recursos y las medidas que se contemplan en las guías y protocolos anti-bullying para los agresores son fundamentalmente de carácter correctivo (Rubio, Díaz \& Cerezo, 2019). Estos instrumentos apenas recogen acciones educativas para el alumnado acosador por lo que el profesorado en ocasiones utiliza recursos diseñados originalmente para resolver conflictos como la mediación y el arbitraje, métodos que no son pertinentes en esta situación (Pulido et al., 2014). Y es que estas estrategias pueden facilitar que se malinterprete el fenómeno del bullying como una confrontación abierta entre iguales del que sale mal parado el más débil, cuando en realidad es lo contrario (Buendía et al., 2015).

La ausencia de recursos que permiten detener la conducta agresora del alumnado acosador desde un enfoque pedagógico pone de manifiesto la conveniencia y necesidad de diseñar instrumentos ad hoc que puedan ser implementados por los profesionales de la educación (Kovač \& Kostøl, 2020). No en vano, por medio del enfoque educativo se obtienen mejores resultados en la detención de las conductas acosadoras del bullying que a través de métodos meramente punitivos (Garandeau et al., 2016).

\section{Factores del bullying y medidas de detención}

Un procedimiento básico que tenga por objeto atajar el bullying cuando ya se ha producido, debe contemplar un conjunto de actuaciones de diferente carácter ya que son varios los factores generales que contribuyen al desarrollo del comportamiento acosador.

La rentabilización y sobreestimación de las conductas de acoso, por ejemplo, es uno de los componentes determinantes en la aparición y mantenimiento de bullying. $\mathrm{Y}$ es que determinados estudiantes obtienen buenos resultados a través de su comportamiento agresivo proactivo, lo que hace que su expectativa de éxito siga vigente (Gini, Pozzoli y Hymel, 2014) y que elija comportarse de forma acosadora en la medida que los beneficios superen los costos personales (Koh \& Wong, 2017; van der Ploeg, Steglich y Veenstra, 2016).

La baja tolerancia a la frustración, también, es otro de los aspectos importantes que favorece que se desencadene la conducta agresiva y la aparición del bullying reactivo (Cole, 1995; Thomas, Connor, \& Scott, 2018). No en vano, algunos estudiantes como resultado de no encontrarse en disposición de obtener algo que desean, como pudiera ser estatus social o éxito académico, proyectan su malestar e impotencia sobre las figuras o arquetipos que creen que representa su anhelo y actúan con represalias sobre ellos (Fung, 2019). Así, el éxito de una parte del alumnado funciona como chivo expiatorio de los fracasos de los agresores que necesitan de una víctima para ejercer su poder o liderazgo (Cerezo, 2009; Morales, Yubero \& Larrañaga, 2016).

Otras de las cuestiones a las que debe responder un instrumento anti-bullying es la baja inteligencia emocional y la incapacidad de reconocer los sentimientos propios y ajenos que pudiera tener este alumnado. $Y$ es que este aspecto favorece que algunos sujetos interpreten el medio escolar en clave dicotómica y que prefieran estar del lado de los agresores antes que ser víctima (Garaigordobil, 2017; Vilariño, Amado \& Alvés., 2013). 
Asimismo, las distorsiones cognitivas durante el procesamiento de información social de una parte del alumnado dan como resultado que tengan una baja comprensión de los estados mentales de las personas con las que tratan, lo que contribuye a que perciban algunas interacciones sociales como una amenaza y respondan de forma agresiva (Crick \& Dodge, 1996; Martinelli et al., 2018).

De esta forma, parece conveniente que el profesorado favorezca por medio de diferentes actividades o procesos metacognitivos que los estudiantes que han agredido reconozcan las conductas de acoso realizadas (Morales, Yubero \& Larrañaga, 2016). Este primer paso favorecería que el alumnado valorase el tipo de agresión que ha cometido, así como el daño que han causado, lo que contribuiría al aumento de la capacidad empática que incide en la disminución de los comportamientos agresivos (Gutiérrez, Escarti \& Pascual, 2011; Hernández-Serrano et al., 2016).

Otra cuestión importante que el profesorado debe potenciar entre el alumnado que han realizado bullying es la atribución causal interna de los acontecimientos, ya que los agresores no suelen responsabilizarse de las conductas acosadoras que han cometido o hacen creer a la víctima que se merece lo que está viviendo (Gairín Sallánez et al., 2012). Por esta razón, las actuaciones con estos chicos y chicas estudiantes deben ir encaminadas a que perciban el coste de respuesta y asuman las sanciones que les corresponden por haber agredido (Gómez et al., 2005; Hubbard et al., 2010).

Por último, y no por ello menos importante, el profesorado que intervenga debe favorecer que el alumnado tenga un estilo de pensamiento avanzado y que su nivel de comprensión sea los más profundo posible (Ritchhart, 2020), ya que esto facilitaría que los estudiantes discriminasen las motivaciones que le han llevado a realizar las agresiones, del propósito que perseguían por medio de ellas. Esto contribuirá a que obtengan una comprensión profunda sobre las verdaderas causas y razones por las que agreden y a advertir que la víctima no es más que un actor neutro al que se le está infligiendo un castigo social, físico y/o psicológico inaceptable.

\section{Niveles de comprensión}

El desempeño que permitiría al alumnado acosador actuar de manera flexible y adaptativa, es decir de forma no acosadora, en las condiciones que le demanda la situación a la que se enfrenta, como son las relaciones sociales con otros chicos y chicas estudiantes en contexto escolar, dependerá del conocimiento profundo o nivel de comprensión que tenga (Perkins, 1999, 2008). Y esto se debe a que las personas aplican y transfieren sus competencias en forma de conocimientos, procedimientos y actitudes a nuevas situaciones de manera progresiva y escalonada (Poblete et al., 2016; Villa \& Poblete, 2007). Asimismo, los diferentes tipos de pensamiento o niveles de comprensión se pueden clasificar en función del tipo de proceso cognitivo que impera en ese desempeño. Y es que los estudiantes al tiempo que son más conscientes de su pensamiento, de las estrategias y del tipo de desempeño que realizan, mejoran sus procesos metacognitivos (Ritchhart, 2020; Ritchhart, Church \& Morrison., 2014; Richhart, Turner \& Hadar, 2009) y su capacidad para explicar, justificar, extrapolar y aplicar el conocimiento en diferentes situaciones (Blythe \& Allen, 2015; Perkins, 2016; Perkins \& Reese, 2014; Perkins \& Tishman, 2011; Stone, 1999). Por ello, en tanto que 
un estudiante alcanza un nivel mayor, obtiene una comprensión más profunda y, por lo tanto, mayor destreza en su desempeño, por lo que resuelve problemas de mayor complejidad y tendrá menores posibilidades de agredir (Véase Figura 1).

\begin{tabular}{ll}
\hline \multicolumn{1}{c}{ Nivel de comprensión } & \multicolumn{1}{c}{ Destrezas de pensamiento } \\
\hline 1. Ingenuo & Práctica intuitiva. \\
2. Novato & Repetición de fórmulas aprendidas. \\
3. Aprendiz & Transferencia de lo aprendido a un contexto diferente. \\
4. Experto & Proactivo. Se adelanta a los acontecimientos. \\
\hline
\end{tabular}

Figura 1. Relación entre niveles y destrezas de pensamiento.

\section{Método}

\section{Objetivos}

El propósito de la investigación que se presenta es diseñar un procedimiento que contribuya a detener las conductas acosadoras del alumnado que realiza bullying. De forma más concreta el estudio pretende lograr los siguientes objetivos específicos:

1. Conocer la incidencia de una intervención compuesta por una secuencia de actividades o procesos metacognitivos en la detención del bullying.

2. Analizar las intervenciones llevadas a cabo con el alumnado acosador en las diferentes sesiones para conocer el nivel de comprensión y tipo de pensamiento por el que pasa el alumnado que realiza bullying, antes de dejar de acosar.

\section{Población y Muestra}

En el curso 2018-2019 en la Comunidad Autónoma Vasca (CAV) se analizaron 755 procesos en Educación Primaria y Educación Secundaria Obligatoria (ESO) por la Inspección del Departamento de Educación, de los que finalmente se identificaron 96 casos como bullying. El estudio que se presenta a continuación se refiere a seis casos, compuesto cada uno de ellos por seis estudiantes victimizados, su respectivos tutores y seis alumnos del primer ciclo de Educación Secundaria Obligatoria (ESO) (14 años) que cometían conductas gravemente perjudiciales a la convivencia y eran susceptibles de ser catalogadas como acoso escolar (Decreto 201/2008, de 2 de diciembre); exclusión y marginación social (no dejar participar); agresión verbal directa (insultos); agresión verbal indirecta (poner motes); agresiones físicas indirectas (esconder objetos o robar); agresiones físicas directas (golpear a alguien); intimidación (obligar a hacer cosas).

Los estudiantes acosadores fueron identificados por medio de los centros educativos de la CAV a los que se les ofreció un servicio de orientación anti-bullying. De esta forma, al principio de la investigación se catalogó el comportamiento de los estudiantes 
agresores como conductas agresivas susceptibles de ser interpretadas como acoso escolar. Esto permitió que se actuase por medio de medidas educativas y no sancionadoras dentro del marco de vías alternativas para la corrección de conductas (decreto 201/2008, de 2 de diciembre). En segundo lugar, se pidió permiso a las familias y tutores legales de estos estudiantes realizar con sus hijas e hijos una actuación psicopedagógica en respuesta a las conductas agresivas probadas que habían cometido.

\section{Instrumento}

Con objeto de medir la percepción de bullying de los estudiantes agresores, de las víctimas, y los tutores que lo habían observado, se utilizó una Escala Visual Analógica (EVA) (Véase Figura 2) (Aitken, 1969). Este instrumento psicométrico que ha sido ampliamente utilizado para medir fenómenos fisiológicos y conductuales (Herdman, Badia \& Sierra, 2001; Van Agt et al., 1994), permitió a los encuestados señalar su grado de acuerdo sobre cada conducta acosadora que se estaba produciendo. De esta forma, tanto el tutor de la víctima, como la propia víctima y el acosador, indicaron una posición por medio de una marca sobre una línea continua que no contenía ni números, ni palabras descriptivas y que en un extremo señalaba poco de acuerdo y en el otro muy de acuerdo (Reips y Funke, 2008). Así el valor de las posiciones que revelaron cada uno de ellos en la línea continua, se obtuvo por medio equivalencias. De esta forma, para una distancia total de $10 \mathrm{~cm}$ de línea, entre 0 y $2 \mathrm{~cm}$ equivalió a un punto, entre 2 y $4 \mathrm{~cm}$ a 2 puntos y así sucesivamente.

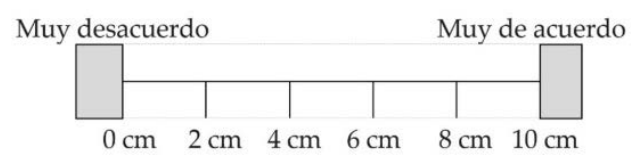

Figura 2. Equivalencias de cm a puntos en un EVA.

Por el contrario, el instrumento que se utilizó para examinar el Nivel de Pensamiento (NP) y el tipo de Procesamiento de la Información (PI) que tenían los estudiantes agresores en cada fase de la intervención fue el registro de las entrevistas participantes (audios) entre cada uno de los acosadores y el educador externo que actuaba. De este modo, se transcribieron y analizaron las seis sesiones de aproximadamente una hora de duración que se produjeron con cada uno de los seis acosadores, en un período de seis semanas.

Para recoger la realidad de lo sucedido en estas intervenciones y valorar los resultados, se documentaron las respuestas de estos estudiantes de forma sistemática atendiendo a criterios de adaptabilidad, variabilidad, gradualidad y pertenencia (Santos, 1990). De esta forma, el gran volumen de datos contextuales que se generó, fue analizado mediante la herramienta informática ATLAS.ti 8.0, lo que permitió codificar y categorizar las respuestas que dieron los acosadores respetando para el análisis de contenido las categorías de los niveles de comprensión (Perkins, 2008). 


\section{Diseño y procedimiento de recogida y análisis de datos}

Para lograr detener las conductas agresivas susceptibles de ser tipificadas como bullying se realizó un estudio de carácter cuantitativo y cualitativo en el contexto del método de investigación-acción de espirales o ciclos (Kolb, 1984; McKernan, 1999) que se compone por cuatro etapas; planificación, implementación, observación y evaluación.

Con el fin de diseñar la secuencia de acciones que pudiese contribuir a detener las conductas bullying que se estaban produciendo, en la primera etapa, se diseñó junto a los tutores y orientadores de cada centro escolar la estrategia que se debía llevar a cabo con cada uno de los estudiantes acosadores. Para ello, teniendo en cuenta los factores generales que originan el bullying y las medidas para su detención señaladas en este trabajo, se planteó un conjunto de intervenciones en base a las diferentes teorías sobre los factores personales que contribuyen a la aparición del bullying, así como a su posible erradicación. Fundamentalmente, en esta primera etapa se planificó para que estos estudiantes realizasen una secuencia de actividades de carácter metacognitivo encaminadas a facilitar el proceso de aprendizaje y reflexión sobre la propia práctica con el fin de hacerle consciente de su proceso, estableciendo líneas claras de mejora que le permitían avanzar en el propio nivel de comprensión y pasar al siguiente (Perkins, 2008).

Concretamente, por medio de un diseño multi-nivelado que se producía en seis fases o sesiones, una por semana, se planificó para que los estudiantes estuviesen en situación de alerta (sesión 1); se responsabilizasen de sus actos (sesión 2); reconociesen que habían acosado (sesión 3); analizasen sus acciones (sesión 4); las evaluasen (sesión 5); y fuesen conscientes del motivo por el que agredían (sesión 6).

Cada una de las seis fases señaladas estaba compuesta por dos sub-fases o procesos de carácter metacognitivo, también denominadas momentos o actividades. Estas intervenciones tenían como fin que los estudiantes acosadores señalasen (actividad 1) y comprendiesen la función del educador que les estaba entrevistando (actividad 2); describen las agresiones perpetradas (actividad 3) y asumiesen el resultado derivado de su acción (actividad 4); asociasen las conductas realizadas con el rol de agresor (actividad 5) y aceptasen que ellos eran los agresores (actividad 6); analizasen el tipo de conducta que habían cometido (actividad 7) y valorasen la gravedad del tipo de conductas que habían realizado (Actividad 8); señalasen (actividad 9) y predijesen el tipo de sanción que corresponden a sus conductas (actividad 10); y por último, con objeto de que estos estudiantes despersonalizasen las agresiones sobre sus víctimas, la dos actividades finales se configuraron para que relacionasen las conductas de acoso realizadas con la finalidad que perseguían (actividad 11) y relacionasen los objetivos del acoso realizados con los motivos que les habían llevado a hacerlo (actividad 12).

En una segunda etapa dentro del método de investigación-acción, con cada uno de los acosadores se implementaron las intervenciones diseñadas y planificadas que se han señalado, de forma metódica y reflexiva. Además, con objeto de conocer el resultado de estas actuaciones, semanalmente, al inicio de cada intervención cada estudiante agresor valoró en una Escala Visual Analógica (EVA) su conducta y talante, cuya respuesta fue cotejada con las observaciones de los profesores y con la percepción de las víctimas.

Tanto víctima, como acosador y tutor manejaron la misma lista de control de conductas acosadoras, que fueron valoradas por medio de esta Escala Visual Analógica 
(EVA). A menor puntuación en este instrumento, mayor percepción sobre la extinción de la conducta acosadora, por lo que estas sólo fueron interpretadas que habían sido detenidas cuando la media de puntos de la víctima, acosador y tutor tuvo una valoración de tres o menos puntos. Asimismo, no se consideró que una conducta de acoso estaba detenida, a pesar de que la media fuese de tres puntos, cuando bien agresor, víctima o tutor valoraron por encima de 3 puntos ese comportamiento específico.

En la tercera etapa denominada observación de esta metodología, con el fin de identificar de forma más profunda la naturaleza de las intervenciones realizadas y el resultado que producía sobre los estudiantes acosadores, se codificaron y categorizaron las respuestas o verbalizaciones que dieron a la secuencia procesos metacognitivos planteado.

Por último, en la cuarta etapa, se evaluó y reflexionó sobre los datos obtenidos y teniendo en cuenta los resultados de la categorización realizada sobre los niveles de comprensión de los estudiantes, se diseñó una propuesta de actuación perfeccionada que queda recogida en este trabajo.

\section{Resultados}

A continuación, se presentan los resultados de la investigación siguiendo los objetivos que se plantearon en el estudio. En relación al primer objetivo (1. Conocer la incidencia de una intervención compuesta por una secuencia de actividades o procesos metacognitivos en la detención del bullying) el análisis de la información obtenida por medio de las escalas visuales analógicas (EVA), pone de manifiesto que los procesos metacognitivos propuestos por el educador en las diferentes intervenciones contribuyen a que los agresores, víctimas y tutores perciban que las conductas de acoso escolar desaparecen paulatinamente (Véase Tabla 1). Es decir, el estudio evidencia que en cada caso a medida que avanzan las intervenciones en las seis semanas, los estudiantes tienden a realizar menos conductas de acoso.

Tabla 1

Evolución de la extinción de las conductas acosadoras

\begin{tabular}{|c|c|c|c|c|c|c|c|c|c|c|c|c|c|c|c|}
\hline \multirow{3}{*}{$\begin{array}{c}\text { Tipo de } \\
\text { bullying }\end{array}$} & \multirow{3}{*}{ Casos } & \multicolumn{14}{|c|}{ Sesiones } \\
\hline & & \multicolumn{2}{|c|}{1} & \multicolumn{2}{|c|}{2} & \multicolumn{2}{|c|}{3} & \multicolumn{2}{|c|}{4} & \multicolumn{2}{|c|}{5} & \multicolumn{2}{|c|}{6} & \multicolumn{2}{|c|}{7} \\
\hline & & $\mathrm{M}$ & DT & $\mathrm{M}$ & DT & M & DT & M & DT & $\mathrm{M}$ & DT & $\mathrm{M}$ & DT & $\mathrm{M}$ & DT \\
\hline \multirow[t]{7}{*}{ Intimidaciones } & 1 & 4,76 & 0,57 & 4,67 & 0,57 & 4,3 & 0,57 & 4 & 1 & 4 & 0 & 3,33 & 0,57 & 2,67 & 0,57 \\
\hline & 2 & 4,33 & 1,15 & 3,67 & 1,15 & 3,33 & 0,57 & 3,33 & 0,57 & 3,33 & 0,57 & 2,67 & 0,57 & 2,67 & 0,57 \\
\hline & 3 & 4,3 & 0,57 & 3,67 & 0,57 & 3,3 & 0,57 & 3 & 1 & 3 & 0 & 2,33 & 0,57 & 2,33 & 0,57 \\
\hline & 4 & 4,3 & 0,57 & 3,33 & 0,57 & 3,3 & 0,57 & 3,67 & 0,57 & 2,33 & 0,57 & 2,33 & 0,57 & 2,67 & 0,57 \\
\hline & 5 & 4,76 & 0,57 & 4 & 0 & 3,3 & 0,57 & 3,3 & 0,57 & 3 & 0 & 2,33 & 0,57 & 2,33 & 0,57 \\
\hline & 6 & 4,33 & 1,15 & 4 & 0 & 3,3 & 0,57 & 2,33 & 0,57 & 2,33 & 0,57 & 2,33 & 0,57 & 2,33 & 0,57 \\
\hline & Total & 4,46 & 0,76 & 3,89 & 0,47 & 3,47 & 0,57 & 3,27 & 0,71 & 2,99 & 0,28 & 2,55 & 0,57 & 2,5 & 0,57 \\
\hline
\end{tabular}




\begin{tabular}{|c|c|c|c|c|c|c|c|c|c|c|c|c|c|c|c|}
\hline \multirow{3}{*}{$\begin{array}{l}\text { Tipo de } \\
\text { bullying }\end{array}$} & \multirow{3}{*}{ Casos } & \multicolumn{14}{|c|}{ Sesiones } \\
\hline & & \multicolumn{2}{|c|}{1} & \multicolumn{2}{|c|}{2} & \multicolumn{2}{|c|}{3} & \multicolumn{2}{|c|}{4} & \multicolumn{2}{|r|}{5} & \multicolumn{2}{|c|}{6} & \multicolumn{2}{|c|}{7} \\
\hline & & $\mathrm{M}$ & DT & $\mathrm{M}$ & DT & M & DT & M & DT & M & DT & $\mathrm{M}$ & DT & M & DT \\
\hline \multirow{7}{*}{$\begin{array}{l}\text { Agresiones } \\
\text { verbales } \\
\text { directas } \\
\text { (Insultos) }\end{array}$} & 1 & 4,3 & 0,57 & 3 & 0 & 3 & 0,57 & 2,67 & 0,57 & 2,67 & 0,57 & 3 & 0,57 & 1,3 & 0,57 \\
\hline & 2 & 4,76 & 0,57 & 4,67 & 0,57 & 3 & 1,15 & 3 & 0,57 & 3 & 0,57 & 2,67 & 0,57 & 1,3 & 0,57 \\
\hline & 3 & 3,67 & 0,57 & 3 & 0,57 & 3 & 0,57 & 2,67 & 0,57 & 3 & 0,57 & 1,3 & 0,57 & 2,67 & 0,57 \\
\hline & 4 & 4 & 0 & 3 & 0 & 3,3 & 0,57 & 3,3 & 0,57 & 3 & 0,57 & 2,33 & 0,57 & 0,57 & 0,57 \\
\hline & 5 & 4,3 & 0,57 & 3 & 0 & 3 & 0 & 3 & 0,57 & 3 & 0,57 & 3 & 0,57 & 3 & 0,57 \\
\hline & 6 & 4 & 0 & 3 & 0 & 3 & 0 & 3 & 0,57 & 3 & 0,57 & 2,67 & 0,57 & 2,67 & 0,57 \\
\hline & Total & 4,17 & 0,38 & 3,27 & 0,19 & 3,05 & 0,47 & 2,94 & 0,57 & 2,94 & 0,57 & 2,49 & 0,57 & 1,91 & 0,57 \\
\hline \multirow{7}{*}{$\begin{array}{l}\text { Agresiones } \\
\text { verbales } \\
\text { indirectas } \\
\text { (motes) }\end{array}$} & 1 & 4 & 1 & 3,67 & 0,57 & 3,3 & 0,57 & 3 & 0 & 2,67 & 0,57 & 2,33 & 1,15 & 1,15 & 2,67 \\
\hline & 2 & 4,76 & 0,57 & 4,3 & 1,15 & 3,3 & 0,57 & 3,6 & 0,57 & 3,67 & 0,57 & 2,33 & 0,57 & 0,57 & 1,3 \\
\hline & 3 & 4,76 & 0,57 & 4,3 & 0,57 & 4 & 0 & 3,3 & 0,57 & 2,67 & 0,57 & 2,33 & 1,15 & 1,15 & 2,67 \\
\hline & 4 & 4,3 & 0,57 & 3,3 & 0,57 & 3,3 & 0,57 & 3,3 & 0,57 & 3 & 0 & 3 & 0 & 0 & 2,67 \\
\hline & 5 & 4,3 & 0,57 & 4,3 & 0,57 & 3,67 & 0,57 & 3,3 & 0,57 & 3,33 & 0,57 & 3,67 & 0,57 & 0,57 & 1,3 \\
\hline & 6 & 5 & 0 & 4,3 & 1,15 & 3,3 & 0,57 & 4 & 1 & 2,67 & 0,57 & 2,33 & 0,57 & 0,57 & 2,67 \\
\hline & Total & 4,52 & 0,54 & 4,02 & 0,76 & 3,47 & 0,47 & 3,41 & 0,54 & 3,00 & 0,475 & 2,66 & 0,66 & 0,66 & 2,21 \\
\hline \multirow{7}{*}{$\begin{array}{l}\text { Exclusión y } \\
\text { marginación } \\
\text { social } \\
\text { (No dejar } \\
\text { participar) }\end{array}$} & 1 & 4 & 0 & 3 & 1 & 4 & 0 & 3 & 0,57 & 2,67 & 0,57 & 2,3 & 1,15 & 2,67 & 0,57 \\
\hline & 2 & 4,3 & 0,57 & 3,3 & 0,57 & 3,67 & 0,57 & 3,3 & 0,57 & 2,67 & 0,57 & 1,67 & 0,57 & 1,67 & 0,57 \\
\hline & 3 & 3,67 & 0,57 & 3,3 & 0,57 & 4,3 & 0,57 & 3,3 & 0 & 1,67 & 0,57 & 2 & 0,57 & 1,67 & 0,57 \\
\hline & 4 & 4,76 & 0,57 & 3,3 & 0,57 & 4,76 & 0,57 & 3,3 & 0,57 & 2,67 & 0,57 & 1,67 & 0,57 & 1,3 & 0,57 \\
\hline & 5 & 4,3 & 0,57 & 3,3 & 0,57 & 4,3 & 0,57 & 3,3 & 0 & 2,67 & 0,57 & 1,67 & 0,57 & 2,67 & 0,57 \\
\hline & 6 & 4,76 & 0,57 & 3,6 & 0,57 & 4,76 & 0,57 & 3,6 & 0 & 3 & 0 & 1,67 & 0,57 & 1,3 & 0,57 \\
\hline & Total & 4,29 & 0,47 & 3,3 & 0,64 & 4,29 & 0,475 & 3,3 & 0,285 & 2,55 & 0,475 & 1,83 & 0,57 & 1,88 & 0,57 \\
\hline \multirow{7}{*}{$\begin{array}{l}\text { Agresiones } \\
\text { físicas directas } \\
\text { (Golpear) }\end{array}$} & 1 & 4,33 & 0,57 & 2,3 & 0,57 & 3 & 0 & 2,3 & 1,15 & 1,3 & 0,57 & 1,67 & 0,57 & 1,3 & 0,57 \\
\hline & 2 & 4 & 1 & 2,3 & 0,57 & 1,3 & 0,57 & 2 & 1 & 1,3 & 0,57 & 1,67 & 0,57 & 1,3 & 0,57 \\
\hline & 3 & 4,76 & 0,57 & 2,3 & 0,57 & 3 & 0 & 2,3 & 1,15 & 2,3 & 0,57 & 1,67 & 0,57 & 1,3 & 0,57 \\
\hline & 4 & 4 & 0 & 2,3 & 0,57 & 3 & 0 & 2 & 1 & 2,3 & 0,57 & 1,67 & 0,57 & 1,3 & 0,57 \\
\hline & 5 & 4 & 0 & 2,3 & 0,57 & 3 & 0 & 2 & 1 & 2,3 & 0,57 & 1,67 & 0,57 & 1,3 & 0,57 \\
\hline & 6 & 4 & 1 & 2,3 & 0,57 & 3 & 0 & 2 & 1 & 1,67 & 0,57 & 1,67 & 0,57 & 1,3 & 0,57 \\
\hline & Total & 4,18 & 0,52 & 2,3 & 0,57 & 2,71 & 0,09 & 2,1 & 1,05 & 1,86 & 0,57 & 1,67 & 0,57 & 1,3 & 0,57 \\
\hline \multirow{7}{*}{$\begin{array}{l}\text { Agresiones } \\
\text { físicas } \\
\text { indirectas } \\
\text { (Esconder, } \\
\text { objetos y robar) }\end{array}$} & 1 & 4 & 1 & 3,3 & 1,58 & 2,3 & 0,57 & 2,3 & 0,57 & 2,3 & 0,57 & 2,67 & 0,57 & 2 & 1 \\
\hline & 2 & 3,67 & 1,15 & 2,67 & 0,57 & 2,3 & 0,57 & 2,3 & 0,57 & 2 & 1 & 2,33 & 0,57 & 2,3 & 1,15 \\
\hline & 3 & 3,3 & 0,57 & 2,67 & 0,57 & 2,3 & 0,57 & 2,3 & 0,57 & 2,67 & 1,52 & 2,33 & 0,57 & 2,2 & 0,57 \\
\hline & 4 & 4,76 & 0,57 & 2,67 & 0,57 & 2,3 & 0,57 & 2,3 & 0,57 & 2,33 & 1,52 & 2,37 & 0,57 & 1,3 & 0,57 \\
\hline & 5 & 3,67 & 0,57 & 2,67 & 0,57 & 2,3 & 0,57 & 2,3 & 0,57 & 2,3 & 0,57 & 2,67 & 0,57 & 2,67 & 0,57 \\
\hline & 6 & 4,33 & 1,15 & 2,67 & 0,57 & 2,3 & 0,57 & 2,3 & 0,57 & 2 & 1 & 2,67 & 0,57 & 1,3 & 0,57 \\
\hline & Total & 3,95 & 0,83 & 2,77 & 0,73 & 2,3 & 0,57 & 2,3 & 0,57 & 2,26 & 1,03 & 2,506 & 0,57 & 1,96 & 0,73 \\
\hline
\end{tabular}

Asimismo, se aprecia que los comportamientos más visibles como las agresiones físicas, se detuvieron antes que las conductas más ocultas o sibilinas, como son las agresiones verbales indirectas y las intimidaciones o comportamientos disruptivos hacia la víctima, por lo que las conductas que más costaron detener fueron las de carácter social y las que se encuentran más arraigadas (Véase Figura 3). 


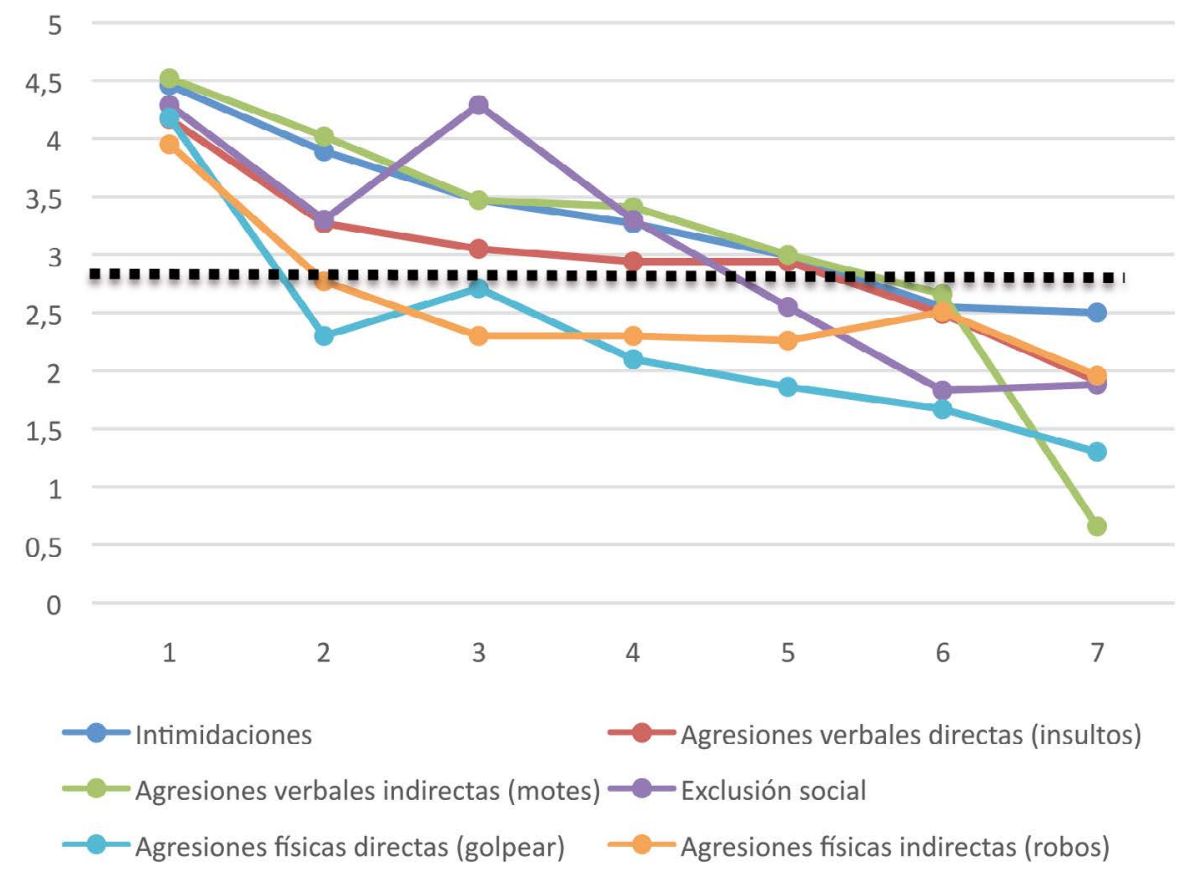

Figura 3. Evolución de las conductas de acoso de carácter físico y material.

Un análisis más concreto pone de manifiesto que a partir de la segunda sesión dejaron de producirse agresiones físicas directas por medio de golpes (M 2,1) y agresiones físicas indirectas (M 2,3). En cambio, la percepción de que las agresiones verbales o insultos (M 2,49), las intimidaciones (M 2,55) y que la exclusión social $(M 1,83)$ ya no se produce $(\leq 3)$ (Véase Figura 3), se aprecia a partir de la cuarta sesión. También queda patente que cuando los estudiantes agresores identificaron el motivo por el que un educador estaba interviniendo con ellos e identificaron la razón por la que estaban siendo entrevistados, todas las conductas disminuyeron considerablemente, siendo la exclusión social la única que repunto.

Asimismo, se observa que el mayor cambio se produjo cuando los estudiantes analizaron el tipo de conducta que estaban cometiendo y valoraron la gravedad de las mismas, a partir de la cuarta sesión (Véase Figura 3).

Los resultados también ponen de manifiesto que los ejercicios de la quinta y sexta fase o sesión, en la que fundamentalmente los estudiantes predijeron las consecuencias de las conductas que estaban realizando y despersonalizaron las agresiones que realizaban sobre la víctima, también favorecieron que el acoso no volviese a aparecer (Véase Figura 3).

En relación al segundo objetivo que persigue el estudio (2. Analizar las intervenciones llevadas a cabo con el alumnado acosador en las diferentes sesiones para conocer el nivel de comprensión y tipo de pensamiento por el que pasa el alumnado que realiza 
bullying, antes de dejar de acosar.) el instrumento de análisis cualitativo ATLAS.ti 8.0, ha permitido identificar el tipo de Procesamiento de la Información (PI) que se produjo en las intervenciones que se llevaron a cabo y el nivel de Compresión o Pensamiento (NP) de los estudiantes, así como el efecto de los procesos metacognitivos en las conductas acosadoras (Véase Figura 4).

De esta forma, los resultados del estudio realizado evidencian que en la primera parte de cada sesión los estudiantes acosadores realizan un tipo de procesamiento de la información que contribuye a consolidar los nuevos aprendizajes y que se ha identificado como la macro categoría asentamiento. También queda de manifiesto que en la segunda parte de cada una de las sesiones el procesamiento de la información es de carácter más elaborado ya que los estudiantes se proyectan e imaginan en el futuro, lo que ha dado lugar a que se clasifique una segunda macro-categoría denominada proyección. Así, la secuencia de procesos metacognitivos secuenciados de menos a más complejidad en cada sesión o momento contribuyen a que los estudiantes agresores asienten o fijen un tipo de procesamiento de la información de carácter básico (macrocategoría asentamiento), y ayuda a que se proyecten y trasciendan hacia un escenario de conductas no acosadoras (macro-categoría proyección).

Del mismo modo, se observa que a medida que avanzan las sesiones se produce un nivel de comprensión diferente en los estudiantes, ya que en las dos primeras fases es de ingenuo, en las dos siguientes de novato y en las dos últimas de aprendiz (Perkins, 1999).

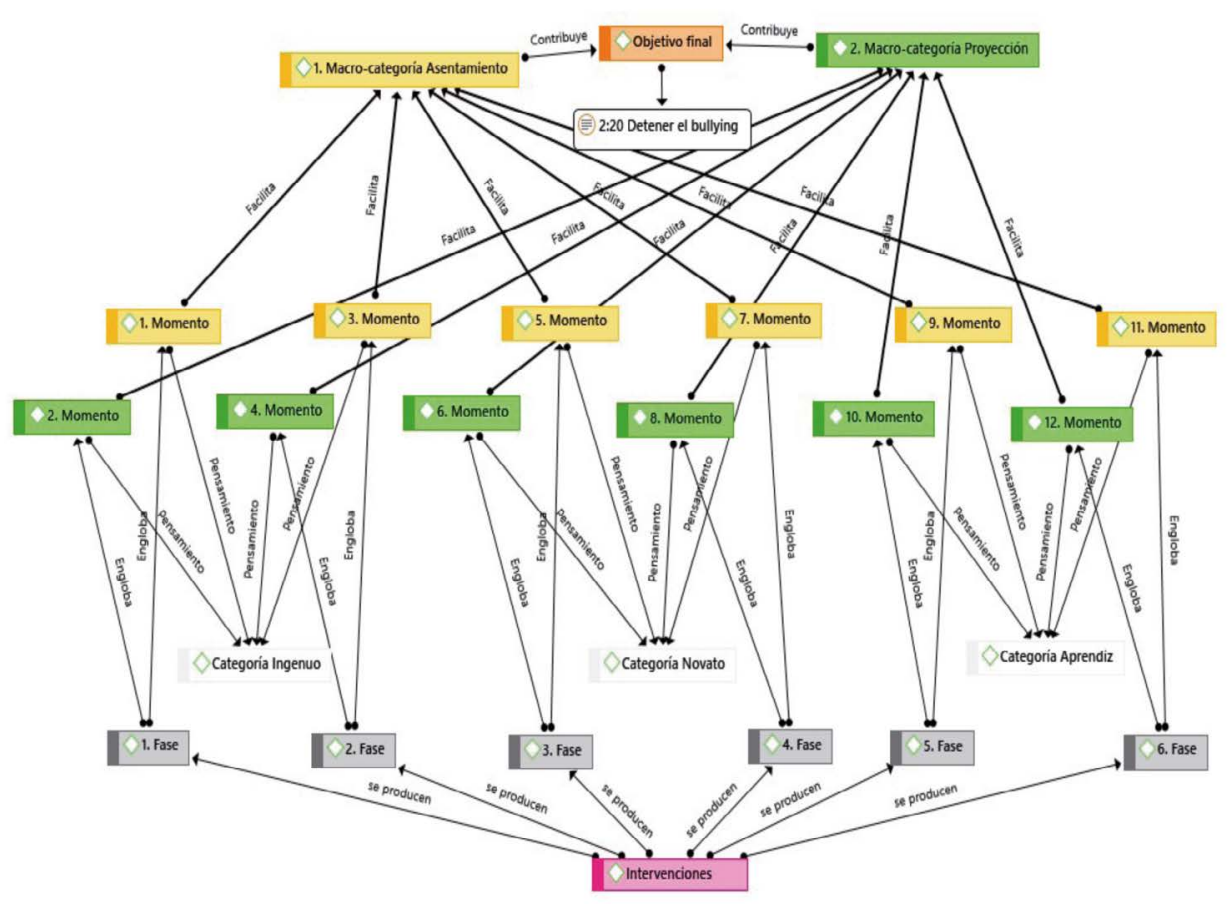

Figura 4. Categorización del efecto de los procesos metacognitivos en las conductas acosadoras. 
Finalmente, el estudio realizado ha dado como resultado una propuesta de actuación anti-bullying perfeccionada en relación a la que se llevó a cabo. Esta queda reflejada (Véase Figura 5) en un procedimiento compuesto por una secuencia de procesos metacognitivos multinivelado en metas y objetivos que contribuyen al desarrollo del Procesamiento de la Información (PI). Esto permite a los estudiantes, en primera instancia, asentar el conocimiento adquirido y posteriormente proyectarse hacia una conducta no acosadora, en la medida que van profundizando en su comprensión o Niveles de Pensamiento (NP).

\begin{tabular}{|c|c|c|c|c|c|}
\hline \multirow[b]{2}{*}{$\begin{array}{l}\text { Fases } \\
\text { Sesiones } \\
\text { Metas }\end{array}$} & \multirow[b]{2}{*}{$\begin{array}{l}\text { Sub-fases } \\
\text { Objetivos }\end{array}$} & \multirow[b]{2}{*}{$\begin{array}{l}\text { Procesos metacognitivos } \\
\text { Secuencia de actividades }\end{array}$} & \multicolumn{2}{|c|}{ PI } & \multirow[b]{2}{*}{ NP } \\
\hline & & & 䒕 & 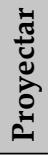 & \\
\hline \multirow{2}{*}{ 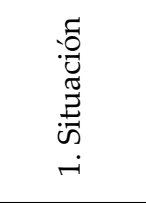 } & $\begin{array}{l}\text { 1.1. Conocer la } \\
\text { función del educador }\end{array}$ & $\begin{array}{l}\text { Los alumnos agresores señalan y describen } \\
\text { quién es la persona con la que está }\end{array}$ & $X$ & & \multirow{4}{*}{ 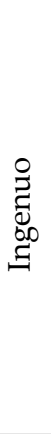 } \\
\hline & $\begin{array}{l}\text { 1.2. Conocer el } \\
\text { motivo de la } \\
\text { situación }\end{array}$ & $\begin{array}{l}\text { Los estudiantes comprenden para qué ha sido } \\
\text { asignado el educador con el que está }\end{array}$ & & $X$ & \\
\hline \multirow{2}{*}{ 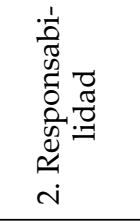 } & $\begin{array}{l}\text { 2.1. Reconocer de las } \\
\text { conductas realizadas }\end{array}$ & $\begin{array}{l}\text { Los acosadores identifican y describen las } \\
\text { agresiones perpetradas. }\end{array}$ & \multicolumn{2}{|l|}{$X$} & \\
\hline & $\begin{array}{l}\text { 2.2. Trascender } \\
\text { sobre las conductas } \\
\text { realizadas }\end{array}$ & $\begin{array}{l}\text { Los alumnos agresores asumen el resultado } \\
\text { derivado de su acción. }\end{array}$ & & $X$ & \\
\hline \multirow{2}{*}{ 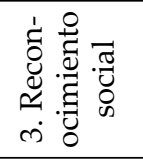 } & $\begin{array}{l}\text { 3.1. Identificar el rol } \\
\text { representado }\end{array}$ & $\begin{array}{l}\text { Los alumnos asocian sus conductas agresivas } \\
\text { genéricas con el rol de agresor. }\end{array}$ & $X$ & & \multirow{4}{*}{$\begin{array}{l}0 \\
\frac{\pi}{\pi} \\
0 \\
7 \\
7\end{array}$} \\
\hline & $\begin{array}{l}\text { 3.2. Reconocerse } \\
\text { como acosador }\end{array}$ & $\begin{array}{l}\text { Los acosadores aceptan el rol representado en } \\
\text { los acontecimientos. }\end{array}$ & & $X$ & \\
\hline \multirow{2}{*}{ 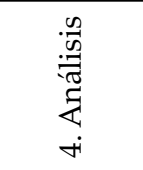 } & $\begin{array}{l}\text { 4.1. Conocer el tipo } \\
\text { de agresión }\end{array}$ & Analiza el tipo de conducta que ha cometido. & \multicolumn{2}{|l|}{$X$} & \\
\hline & $\begin{array}{l}\text { 4.2. Valorar el tipo de } \\
\text { agresión }\end{array}$ & $\begin{array}{l}\text { Los estudiantes valoran la gravedad del tipo } \\
\text { de conductas que ha realizado }\end{array}$ & & $X$ & \\
\hline \multirow{2}{*}{ 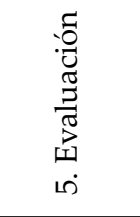 } & $\begin{array}{l}\text { 5.1. Comprender las } \\
\text { consecuencias }\end{array}$ & $\begin{array}{l}\text { Los estudiantes agresores señalan que el resul- } \\
\text { tado de su conducta suele tener una sanción. }\end{array}$ & $X$ & & \multirow{4}{*}{$\begin{array}{l}\text { N } \\
\frac{N}{0} \\
\frac{0}{2} \\
\frac{2}{2}\end{array}$} \\
\hline & $\begin{array}{l}\text { 5.2. Evaluar las } \\
\text { consecuencias que } \\
\text { tienen hacia él }\end{array}$ & $\begin{array}{l}\text { Los acosadores predicen las consecuencias de } \\
\text { sus acciones acosadoras }\end{array}$ & & $X$ & \\
\hline \multirow{2}{*}{ 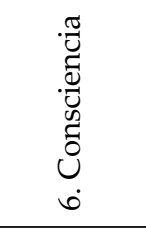 } & $\begin{array}{l}\text { 6.1. Analizar el } \\
\text { objetivo del acoso }\end{array}$ & $\begin{array}{l}\text { Los alumnos agresores relacionan las conduc- } \\
\text { tas de acoso realizadas con la finalidad que } \\
\text { persiguen. }\end{array}$ & \multicolumn{2}{|l|}{$x$} & \\
\hline & $\begin{array}{l}\text { 6.2. Ser consciente } \\
\text { del motivo por el que } \\
\text { acosa }\end{array}$ & $\begin{array}{l}\text { Los alumnos agresores relacionan los objeti- } \\
\text { vos del acoso realizado con los motivos que le } \\
\text { han llevado a hacerlo. }\end{array}$ & & $X$ & \\
\hline
\end{tabular}

Figura 5. Procedimiento para detener el bullying. 


\section{Discusión y conclusiones}

Una vez examinados los resultados de las diferentes intervenciones con los seis alumnos acosadores, se puede concluir que se ha identificado un modelo de actuación estándar que puede ser implementado para detener las conductas de acoso escolar en el momento en que se están produciendo.

En la primera sesión de la intervención las conductas acosadoras de los estudiantes se detuvieron de forma momentánea. El hecho de que un sujeto adulto externo a la institución educativa actuase por las conductas agresivas que se están produciendo predispuso a los estudiantes a una situación de alerta.

Los procesos metacognitivos que se realizaron en el contexto de la segunda sesión y que tenían por objeto desarrollar la atribución causal interna de los acontecimientos para extinguir las conductas agresivas, sólo lograron que se detuvieran las relacionadas con las agresiones físicas directas y los robos. Los comportamientos acosadores más arraigados y de carácter más social necesitaron de mayor tratamiento o trabajo (Hubbard, et al., 2010).

El mayor cambio por parte de los estudiantes se observó a partir de la tercera y cuarta sesión, cuando los estudiantes agresores asumieron su responsabilidad y valoraron la gravedad de sus conductas. En el momento en que se trabajaron las habilidades de pensamiento de tipo causal, alternativo y consecuencial, tanto agresores, víctimas como tutores valoraron que se producían menos conductas acosadoras (Segura, 2005).

Las intervenciones de la quinta sesión facilitaron a que se mantuviesen extinguidas las conductas que habían desaparecido. Y es que trabajar sobre el sentido de las consecuencias y sobre el perjuicio que tiene romper las normas de convivencia contribuyen a no agredir (Chaux et al., 2016, Gázquez, Pérez y Carrión, 2011).

Por último, el hecho de que los estudiantes agresores en la sexta sesión no encontrasen ninguna razón objetiva para acosar a sus víctimas, facilitó que estos despersonalizasen los motivos por los que agredían a sus víctimas.

De la investigación realizada también se concluye que en caso de que no se alcancen los objetivos y metas multinivelados establecidos o programados para alguna sesión, esta tiene que repetirse. Además, la periodicidad de las intervenciones, así como el tipo de proceso metacognitivo que se proponga en cada situación o caso, debe ajustarse a cada estudiante.

Otro aspecto relevante del estudio es que los estudiantes por medio de los procesos metacognitivos estructurados realizan dos tipos de procesamiento de la información diferente. El primer tipo facilita al estudiante acosador la comprensión y asentamiento de ideas coherentes y básicas relacionadas con la función que tiene el educador externo que va a intervenir con él, el reconocimiento de las conductas que ha realizado, la identificación del rol representado, así como del tipo de agresión que ha llevado a cabo dentro del marco de derechos y deberes que establece el sistema educativo, la comprensión de las consecuencias de las mismas y el objetivo o meta que persigue el acoso que ha llevado a cabo. 
El segundo tipo de procesamiento de la información que han desarrollado el alumnado acosador, por el contrario, es de carácter más abstracto y complejo, y les permite trascender y progresar hacia una conducta menos acosadora. En la medida que identifican la causa que les ha llevado a estar en esa situación, asumen las conductas que han realizado, se reconocen como sujetos acosadores, valoran el tipo de agresión que han perpetrado, la evalúan y disciernen el motivo por el que han agredido, despersonalizan la conducta acosadora de su víctima.

También se han reconocido diferentes niveles de comprensión en las diferentes sesiones o momentos (Perkins, 2008, 2016). Los estudiantes acosadores a medida que avanzaron y progresaron, adquirieron un desempeño más flexible y elaborado, lo que les permitió actuar de forma más consciente y en consecuencia menos acosadora.

En este sentido se concluye que diseñar procesos metacognitivos orientados a que los estudiantes acosadores desarrollen dos tipos de procesamiento de la información de forma recurrente y cíclica, les permite empoderarse progresivamente y facilita a que accedan a niveles de comprensión de rango superior, lo que incide sobre algunos de los factores de riesgo más significativos que produce el acoso escolar. Por ello sería interesante que las futuras líneas de investigación se orientasen hacia la personalización del diseño de recursos que permitan intervenir con los estudiantes acosadores en base a la motivación que les lleva a realizar este tipo de conductas.

\section{Financiado}

Proyecto de investigación realizado bajo el amparo del grupo consolidado (IT119519) del Sistema Vasco Universitario, Departamento de Educación, Universidades e Investigación - Gobierno Vasco, España.

\section{Referencias}

Aitken, R. B. C. (1969). Measurements of feelings using visual analogue scales. Proc. $R$ Soc Med, 62, 989-993.

Blythe, T. \& Allen, D. (2015). Looking Together at Student Work, Third Edition. Teachers College Press.

Buendía, L., Expósito, J., Aguadez, E.M., \& Sánchez, C.A. (2015). Análisis de la convivencia escolar en las aulas multiculturales de Educación Secundaria. Revista de Investigación Educativa, 33(2), 303-319. http://dx.doi.org/10.6018/rie.33.2.211491

C. Bonell, E. Allen, E. Warren et al. (2018). Effects of the Learning Together intervention on bullying and aggression in English secondary schools (INCLUSIVE): a cluster randomised controlled trial. The Lancet, 392, 2452-2464. https://doi.org/10.1016/ S0140-6736(18)31782-3

Cerezo, F. (2009). Bullying: Análisis de la situación en las aulas españolas. International Journal of Psychology and Psychological Therapy, 9(3), 383-395.

Cerezo, F., Sánchez, C., Ruiz, C., \& Arense, J.J. (2015). Roles en bullying de adolescentes y preadolescentes, y su relación con el clima social y los estilos educativos parentales. Revista de Psicodidáctica, 20(1), 139-155. 
Chaux, E. Velazquez, A.M., Schultze-krumbhoiz, A., \& Scheithamer, H. (2016). Effects of the cyberbullying prevention program Media Heroes (Medienhelden) on traditional bullying. Aggressive Behavior, 42, 157-165. https://doi.org/10.1002/ab.21637

Cole, E. (1995). Responding to School Violence: Understanding Today for Tomorrow. Canadian Journal of School Psychology, 11(2), 108-116. https://doi. org/10.1177/082957359601100213

Crick, N., \& Dodge, K. (1996). Social Information-Processing Mechanisms in Reactive and Proactive Aggression. Child Development, 67(3), 993-1002.

Decreto 201/2008, de 2 de diciembre, sobre los derechos y deberes de los alumnos y alumnas de los centros docentes no universitarios de la Comunidad Autónoma del País Vasco.

Della Cioppa, V., O’Neil, A., \& Craig, W. (2015). Learning from traditional bullying interventions: A review of research on cyberbullying and best practice. Aggression and Violent Behavior, 23, 61-68. https://doi.org/10.1016/j.avb.2015.05.009

Gairín Sallánez, J., Armengol Asparó, C., \& Silva García, B. (2012). El bullying escolar. Consideraciones organizativas y estrategias para la intervención. Educación XX1, 16(1). https://doi.org/10.5944/educxx1.16.1.715

Garaigordobil, M. (2017). Conducta antisocial: conexión con bullying/cyberbullying y estrategias de resolución de conflictos. Psychosocial Intervention, 26(1), 47-54. https://dx.doi.org/10.1016/j.psi.2015.12.002

Garandeau, C. F., Vartio, A., Poskiparta, E., \& Salmivalli, C. (2016). School bullies' intention to change behavior following teacher interventions: Effects of empathy arousal, condemning of bullying, and blaming of the perpetrator. Prevention Science, 17(8), 1034-1043. https://doi.org/10.1007/s11121-016-0712-x

Gázquez, J. J., Pérez, M. C., \& Carrión, J. J. (2011). Clima escolar y resolución de conflictos según el alumnado: Un estudio europeo. Revista de Psicodidáctica, 16, 39-58.

Gini, Pozzoli \& Hymel. (2014). Moral disengagement among children and youth: A meta-analytic review of links to aggressive behavior. Aggressive Behavior, 40, 56-68. http://dx.doi.org/10.1002/ab.21502

Gómez, A., Gala, FJ., Lupiani, M., Bernalte, A., Miret, MT., Lupiani, S., \& Barreto, MC. (2005). El bullying y otras formas de violencia adolescente. Cuad Med Forense, 13(48-49), 165-177.

Granero, A., \& Manzano, A. (2018). Posibilidades del programa KiVa para hacer frente al bullying homofóbico y transfóbico. Revista Complutense De Educación, 29(4), 943-958. https://doi.org/10.5209/RCED.54346

Gutiérrez, M. Escartí, A., \& Pascual, C. (2011). Relaciones entre empatía, conducta prosocial, agresividad, autoeficacia y responsabilidad personal y social de los escolares. Psicothema, 23(1), 13-19.

Herdman, M., Badía, X., \& Berra, S. (2001). El EuroQol-5D: una alternativa sencilla para la medición de la calidad de vida relacionada con la salud en atención primaria. Atención primaria, 28(6), 425-429.

Hernández-Serrano, O., Espada, J. P., \& Guillén-Riquelme, A. (2016). Relationship of the prosocial behaviour, the problem-solving skills and the use of drugs amongst adolescents. Anales De PsicologíalAnnals of Psychology, 32(2), 609-616. https://doi. org/10.6018/analesps.32.2.204941 
Hubbard, J. A., McAuliffe, M.D., Morrow, M.T., \& Romano, L.J. (2010). Reactive and proactive aggression in childhood and adolescence: Precursors, outcomes, processes, experiences and measurement. Journal of Personality, 78, 95-118. https://doi. org/10.1111/j.1467-6494.2009.00610.x

Koh, J.-B., \& Wong, J. S. (2015). Survival of the fittest and sexiest: Evolutionary origins of adolescent bullying. Journal of Interpersonal Violence. 32(17), 2668-2690. https:// doi.org/10.1177/0886260515593546

Kolb, D. (1984). Experiental Learning: Experience as the Source of Learning and Development. Prentice Hall.

Kovač, V.B., \& Kostøl, E.M.F. (2020). Helping children in bullying situations: The role of intersubjective understanding and co-regulation. School Psychology International.41(3), 295-309. https://doi.org/10.1177/0143034320903789

Lyng, S.T. (2018). The Social Production of Bullying: Expanding the Repertoire of Approaches to Group Dynamics. Children \& Society, 32(6), (492-502). https://doi. org/10.1111/chso.12281

Martinelli, A., Ackermann, K., Bernhard, A., Freitag, C.M., \& Schwenck, C. (2018). Hostile attribution bias and aggression in children and adolescents: a systematic literature review on the influence of aggression subtype and gender. Aggression and Violent Behavior, 39, 25-32.

McKernan, J. (1999). Investigación-acción y currículum. Morata.

Morales, J.F., Yubero, S., \& Larrañaga, E. (2016). Gender and bullying: Application of a three-factor model of gender stereotyping. Sex Roles, 74(3-4), 169-180. https:// doi.org/10.1007/s11199-015-0463-3

Mytton, J.A., DiGuiseppi, C., Gough, D., \& Taylor, R.S., Logan. (2006). S. School-based secondary prevention programmes for preventing violence. Cochrane Database of Systematic Reviews. 3:CD004606. https://doi.org/10.1002/14651858.CD004606.pub2

Nocentini, A., \& Menesini, E. (2016). KiVa Anti-Bullying Program in Italy: Evidence of Effectiveness in a Randomized Control Trial. Prevention Science, 17, 1012-1023. https://doi.org/10.1007/s11121-016-0690-z

Olweus, D. (2013). School bullying: Development and some important challenges. Annual Review of Clinical Psychology, 9, 1-14. https://doi.org/10.1146/annurevclinpsy-050212-185516

Perkins, D. (1999). ¿Qué es la comprensión? En M. Stone, La Enseñanza para la Comprensión (pp. 69-95). Paidós.

Perkins, D (2008). La escuela inteligente. Gedisa

Perkins, D. (2016). Educar para un mundo cambiante ¿Qué necesitan aprender realmente los alumnos para el futuro? SM.

Perkins, D. N., \& Reese, J. D. (2014). When change has legs. Educational Leadership, 71(8), 42-47.

Perkins, D., \& Tishman, S. (2011). El Lenguaje del Pensamiento. Educadores: Revista de renovación pedagógica, 236, 46-58.

Poblete, M., Bezanilla, M.J., Fernández-Nogueira, D., \& Campo, L. (2016). Formación del docente en competencias genéricas: Un instrumento para su planificación y desarrollo. Revista Educar, 52(1), 71-91. https://doi.org/10.5565/rev/educar.713 
Pulido, R., Calderón, S., Martín-Seoane, G., \& Lucas-Molina, B. (2014). Implementación de un programa de mediación escolar: análisis de las dificultades percibidas y propuestas de mejora. Revista Complutense de Educación, 25(2), 375-392. https:// doi.org/10.5209/rev_RCED.2014.v25.n2.41610

Reips, U., \& Funke, F. (2008). Interval-level measurement with visual analogue scales in Internet-based research: VAS Generator. Behavior Research Methods, 40, 699-704. https://doi.org/10.3758/BRM.40.3.699

Ritchhart, R. (2020). The Power of Making Thinking Visible: Practices to Engage and Empower All Learners. Services, Jossey-Bass.

Ritchhart, R., Church, M., \& Morrison, K. 2014). Hacer visible el pensamiento: cómo promover el compromiso, la comprensión y la autonomía de los estudiantes. Paidós.

Ritchhart, R., Turner, T., \& Hadar, L. (2009). Uncovering students' thinking about thinking using concept maps. Metacognition and Learning, 4(2), 145-159.

Rubio, F.J., Díaz, A., \& Cerezo, F. (2019). Bullying y cyberbullying: la respuesta de las comunidades autónomas. Revista Electrónica Interuniversitaria de Formación del Profesorado, 22(1), 145-157. http://dx.doi.org/10.6018/reifop.22.1.332311

Ruiz, R., Riuró, M., \& Tesouro, M. (2015). Estudio del bullying en el ciclo superior de primaria. Educación XXI, 18(1), 345-368. https://doi.org/10.5944/educxx1.18.1.12384

Saarento, S., Boulton, A., \& Salmivalli, C. (2015). Reducing bullying and victimization: Student- and classroom-level mechanisms of change. Journal of Abnormal Child Psychology, 43, 61-76.

Santos, M. A. (1990). Hacer visible lo cotidiano. Akal.

Segura, M. (2005). Enseñar a convivir no es tan difícil. Desclée de Brouwer.

Smith, P. (2016). Bullying: Definition, types, causes, consequences and intervention. Social and Personality Psychology Compass, 10(9), 519-532

Stone, M. (1999). ¿Qué es la Enseñanza para la Comprensión? En M. Stone. La Enseñanza para la comprension. Vinculacion entre la investigacion y la práctica (pp. 95-96). Paidós.

Swearer, S,M., \& Hymel, S. (2015). Understanding the psychology of bullying: Moving toward a social-ecological diathesis-stress model. Am Psychol, 70(4), 344-53. https:// doi.org/10.1037/a0038929

Thomas, H.J., Connor, J.P., \& Scott, J.G. (2018). Why do children and adolescents bully their peers? A critical review of key theoretical frameworks. Soc Psychiatry Psychiatr Epidemiol 53, 437-451. https://doi.org/10.1007/s00127-017-1462-1

Van Agt, H. M., Essink-Bot, M.L., Krabbe, P.F., \& Bonsel, G.J. (1994). Test-retest reliability of health state valuations collected with the EuroQol questionnaire. Social science $\mathcal{E}$ medicine, 39(11), 1537-1544.

Van der Ploeg, R., Steglich, C., \& Veenstra, R. (2016). The support group approach in the Dutch KiVa anti-bullying programme: Effects on victimisation, defending and well-being at school. Educational Research, 58(3) (2016), 221-236. https://doi. org/10.1080/00131881.2016.1184949

Vilariño, M., Amado, B. G., \& Alves, C. (2013). Menores infractores: un estudio de campo de los factores de riesgo. Anuario de Psicología Jurídica, 23, 39-45. https:// doi.org/10.5093/aj2013a7 
Villa, A., \& Poblete, M. (2007). Aprendizaje basado en competencias. Una propuesta para la evaluación de las competencias genéricas. Mensajero.

Volk, A. A., Dane, A. V., \& Marini, Z. A. (2014). What is bullying? A theoretical redefinition. Developmental Review, 34, 327-343.

Fecha de recepción: 11 de abril de 2020.

Fecha de revisión: 27 de abril de 2020.

Fecha de aceptación: 17 de noviembre de 2020. 